\title{
Implementation of Gray Image Encryption using Multi- Level of Permutation and Substitution
}

\author{
Dina Riadh Alshibani \\ Assistant Lecturer \\ Computer Science Department \\ University of Al Mustansiriyah / Iraq - Baghdad
}

\author{
Rasha Shaker Ibrahim \\ Assistant Chief Programmer \\ Computer Science Department \\ University of Al Mustansiriyah / Iraq - Baghdad
}

\begin{abstract}
In this paper a new Gray image encryption system is presented. It is based on permutation and substitution of image pixels using secret keys in time domain. The system is with multilevel to increase the security and to present an encrypted image with low pixel correlation, high entropy and uniform distributed histogram. The Tinkerbell map, Zaslavsky Map and Arnold Transform are employed in keys generation to be used in the permutation and substitution process. Test results are done with definite investigation to show that the proposed image encryption is very secure because of its vast key space and robust permutation-diffusion mechanism.
\end{abstract}

\section{General Terms}

Security, Image Processing.

\section{Keywords}

Choatic maps, Arnold Transform, Tinkerbell map, Zaslavsky Map, Permutation, Subsitusion.

\section{INTRODUCTION}

In the course of recent decades, chaos has been discovered to be exceptionally helpful and to have extraordinary potential in diverse fields of physics, mathematics, engineering, biology, chemistry, and financial matters. Exactly toward the begin of a decade ago, the persistent and additionally discrete chaotic dynamical systems have been utilized for the advancement of cryptosystems.

The reason of applying chaos theory in cryptography lies in its intrinsic features. These properties of chaos includes: affectability to modest changes in introductory conditions, random-like manners, ergodicity, unstable periodic orbits, desired diffusion and confusion properties, and one-way property. Due to these properties, chaotic systems have become a very good candidate for use in the field of cryptography $[1,2,3]$.

\section{CHOATIC MAP}

Brief descriptions for three types of the chaotic maps (Tinkerbell map, Zaslavsky Map and Arnold Transform) that are used in this paper are introduced in the following section.

\subsection{Tinkerbell Map}

The Tinkerbell map is a 2-D discrete-time dynamical system, and is defined as:

$$
\begin{gathered}
X_{n+1}=X_{n}^{2}-Y_{n}^{2}+a X_{n}+b Y_{n} \ldots 1 \\
Y_{n+1}=2 * X_{n} * Y_{n}+c X_{n}+d Y_{n} \ldots 2
\end{gathered}
$$

Where $X_{n}, Y_{n}$ are current chaotic values and $X_{n+1}, Y_{n+1}$, are next chaotic values and $a, b, c, d$ are control parameters. Details of Tinkerbell map can be found in [4]. The key set for
Tinkerbell map is $\left\{\mathrm{X}_{0}, \mathrm{Y}_{0}, \mathrm{a}, \mathrm{b}, \mathrm{c}, \mathrm{d}\right\}$. Commonly used initial values and parameters are $a=0.9, b=-0.6013, c=2.0, d=0.50$.

\subsection{Zaslavsky Map}

Zaslavsky map is a discrete-time dynamical system, and is defined as:

$$
\begin{aligned}
& \mathrm{x}_{\mathrm{n}+1}=\bmod \left(\mathrm{x}_{\mathrm{n}}+\mathrm{v}\left(1+\mu \mathrm{y}_{\mathrm{n}}\right)+\varepsilon \mathrm{v} \cos \left(2 \pi \mathrm{x}_{\mathrm{n}}\right), 1\right) \ldots .3 \\
& \mathrm{y}_{\mathrm{n}}=\mathrm{e}^{-\tau}\left(\mathrm{y}_{\mathrm{n}}\right)+\varepsilon \cos \left(2 \pi \mathrm{x}_{\mathrm{n}}\right) \ldots .4 \\
& \text { And } \mu=\frac{1-\mathrm{e}^{-\tau}}{\tau}
\end{aligned}
$$

Where $v, \tau, \varepsilon$ are control parameters and e is exponentiation. The key set for Zaslavsky map is

$\left\{\mathrm{x}_{0}, \mathrm{y}_{0}, \mathrm{v}, \tau, \varepsilon\right\}$. Commonly used values for the parameters are $\quad \mathrm{v}=12.6695, \varepsilon=9.1, \tau=3.0$. Details of Zaslavsky map can be found in [5].

\subsection{Arnold Transform}

Arnold transform is chosen as pre-treatment method for open image as it is simple and periodic. Therefore, the scrambling transform gives a secondary security for the digital products. In addition, after scrambling transform, the spatial relationships of the pixels of an image has been destroyed, which makes it evenly distributed in all the space, so the robustness of the algorithm was improved in this way. The discrete Arnold transformation is defined as follows:

$$
\left[\begin{array}{c}
X n+1 \\
Y n+1
\end{array}\right]=\left[\begin{array}{cc}
1 & a \\
b & a+b
\end{array}\right]\left[\begin{array}{l}
X n \\
Y n
\end{array}\right]=A\left[\begin{array}{l}
X n \\
Y n
\end{array}\right] \bmod N \quad \ldots .5
$$

Where, $(\mathrm{x}, \mathrm{y})=\{0,1, \ldots . \mathrm{N}\}$ are pixel coordinates from original image. $\left(x_{n+1}, y_{n+1}\right)$ are corresponding results after Arnold Transform, $\mathrm{a}$ and $\mathrm{b}$ positive integers, $\operatorname{det}(\mathrm{A})=1[6]$.

\section{THE PROPOSED SYSTEM}

The Proposed Image Encryption Algorithm is for the most part comprises of two stages. So as to dispose of the relationship of image pixels in closest region the pixel positions is blended this present the first stage. The second stage will be performed by change of pixel dark qualities. Fig. 1 demonstrates the general structure of the proposed image encryption and unscrambling calculation. The base line of the proposed image encryption system can be summarized as follows:

1. Read a gray scale image (img) of size $\mathrm{N}^{*} \mathrm{~N}$

2. Mixing stage ( first level):

- Generation of Transformation key1 and key2. 
- Transform the image columns with the Transformation key1.

- Transform the image rows with the Transformation key2.

- Apply Arnold Transform.

- Bit pixel permutation.

3. Confusion stage (second level):

- Generation of mask key.

- Application of XOR operation between mask key and image pixels resulted from first level.

The general structure of the proposed image decryption design can be summarized as follows:

1. Read the encrypted gray scale image (Eimg) of size $\mathrm{N} * \mathrm{~N}$.

2. Inverse Confusion stage:

- Generation of mask key.

- Application of XOR operation between mask key and image pixels.

3. Inverse Mixing stage:

- Inverse bit pixel permutation

- Apply inverse Arnold Transform.

- Generation of Transformation key1 and Transformation key2.

- Transform the rows of the transformed image with the Transformation key2.

- Transform the columns of the transformed image with the Transformation key1.

\subsection{Mixing Stage}

The motivation behind shuffling stage is to diminish the high connection between neighboring pixels in the plain image. Let I be a gray original image of size $\mathrm{N}^{*} \mathrm{~N}$, it is a matrix containing $\mathrm{N}$ rows and $\mathrm{N}$ columns, and the gray values ranges from 0 to 255 . In the process of permutation the first step in this work is to create the necessary keys for scrambling the image data.

Firstly N/2 chaotic values $\left\{\left(x \operatorname{tin}_{1}, x \operatorname{tin}_{2}, \ldots, x t i n_{N / 2}\right)\right.$, $\left(\right.$ tin $\left._{1}, y \operatorname{tin}_{2}, \ldots, y \operatorname{tin}_{N / 2}\right)$

$\left.\left.\left(x_{z a s_{1}}, \operatorname{xzas}_{2}, \ldots, x_{z a s_{N / 2}}\right) y_{z a s_{1}},{y z a s_{2}}_{2}, \ldots, y_{z a s_{N / 2}}\right)\right\}$ are initiated by using Eq. $(1,2,3,4)$, then chaotic values will be cross-coupled to form one matrix $\mathrm{M}$ of size $\mathrm{N}+\mathrm{N}$ as shown in the Fig. 2. Then the First $\mathrm{N}$ of matrix $\mathrm{M}$ will be transcribed in $P e r_{-} C$ and the second $\mathrm{N}$ in Per_R. Per_C And Per $R$ are arranged, and the positions of sorted chaotic values in the original chaotic sequence are found and stored in $P e r_{-} C^{\prime}$ and $P e r_{-} R^{\prime}$. The next step is to scramble column position of all values from first row to last row according to $P e r_{-} C^{\prime}$. Similarly scramble row position of all values from first column to last column according to $P e r_{-} R^{\prime}$. This will form the first and second steps of the mixing stage.

The third step of mixing stage is applying Arnold Transform to the transformed image. The steps of applying Arnold Transform can be summarized as follows:
Step 1: Partition the transformed image $I_{T r}(r, c)$ into $8 \times 8$ size blocks, $b l k_{1}, b l k_{2}, \ldots, b l k_{n}$.

Step 2: Apply Arnold Transform within block $B_{i}$ to rearrange the pixels of the block, where $i=1,2,3, \ldots, n$.

Step 3: Apply Arnold Transform within whole image $I 1_{T r}(r, c)$ to rearrange the pixels.After repeating this step for $\mathrm{n}$ times shuffled image $\mathrm{S}(\mathrm{x}, \mathrm{y})$ is finally produced.

The fourth step of the mixing stage is the bit pixel permutation. The image can be seen as an array of pixels, each with eight bits for 256 gray levels. In the bit permutation technique the bits in each pixel taken from the image are permuted. The entire array of these permuted pixels forms the encrypted image. Suppose the current pixel value is 65 convert it to its binary presentation then rotate only one bit to the left. After bit pixel permutation the pixel value will be 192. Bit pixel permutation used in this paper is shown in Fig. 3. This stage shuffles all pixels and de-correlates the neighboring pixels

\subsection{Substitution Stage}

As a start, generate $(\mathrm{N} \times \mathrm{N} \times 8) / 2$ chaotic values of xtin, ytin, xzas, yzas the generated chaotic series which are a factual valued. These factual valued cross coupled into two matrix mask_key 1 and mask_key2 of size $(\mathrm{N} \times \mathrm{N} \times 8)$ for each as shown in the Figure 2. mask_key1 And mask_key2 can be transformed into binary series as following:

$$
\begin{aligned}
\operatorname{ser} 1_{\mathrm{i}, 1} & =\operatorname{Round}\left(\operatorname{Abs}\left(\text { mask_key }_{i, 1} \times 10^{14}\right)\right) \bmod 2 \ldots .6 \\
\operatorname{ser} 2_{\mathrm{i}, 1} & =\operatorname{Round}\left(\operatorname{Abs}\left(\text { mask_key }_{i, 1} \times 10^{14}\right)\right) \bmod 2 . .7
\end{aligned}
$$

Each 8 consecutive bit of $\operatorname{ser} 1_{i, 1}$ and ser $2_{i, 1}$ will be converted into integer value after xored with the following 8 consecutive bit in $\operatorname{ser} 1_{i, 1}$ and $\operatorname{ser} 2_{i, 1}$. Reshape integer sequence stored in arrayser $1_{\mathrm{i}, 1}$ and $\operatorname{ser} 2_{\mathrm{i}, 1}$ into a two dimensional matrix Mask1 $(\mathrm{N} * \mathrm{~N})$ and Mask2(N*N). Finally, Mask1 and Mask2 are used to form the Mask in which each pixel of Shuffled image $[i, j]$ is xored with corresponding value of Mask [ $i, j]$.

Masking operation is performed by following formula:

$$
\text { enc }_{\text {img(i,j) }}=\left(\left(\operatorname{shu}_{i m g(i, j)} \otimes \operatorname{Mask}(\mathrm{i}, \mathrm{j})\right) \otimes \operatorname{shu}_{i m g(i-1, j)}\right) . .9
$$

The decryption process similar to the encryption one, described above, but in the reverse order.

The following matlab function is used to illustrate how to mix the two masks (Mask1 and Mask2) into one mask (Mask).

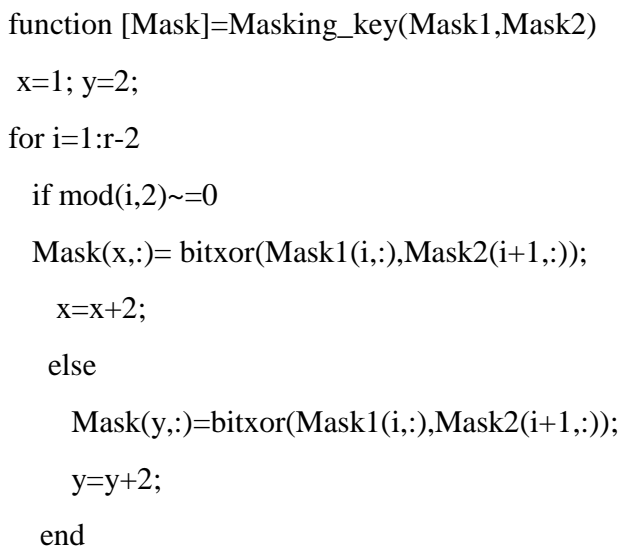

end 


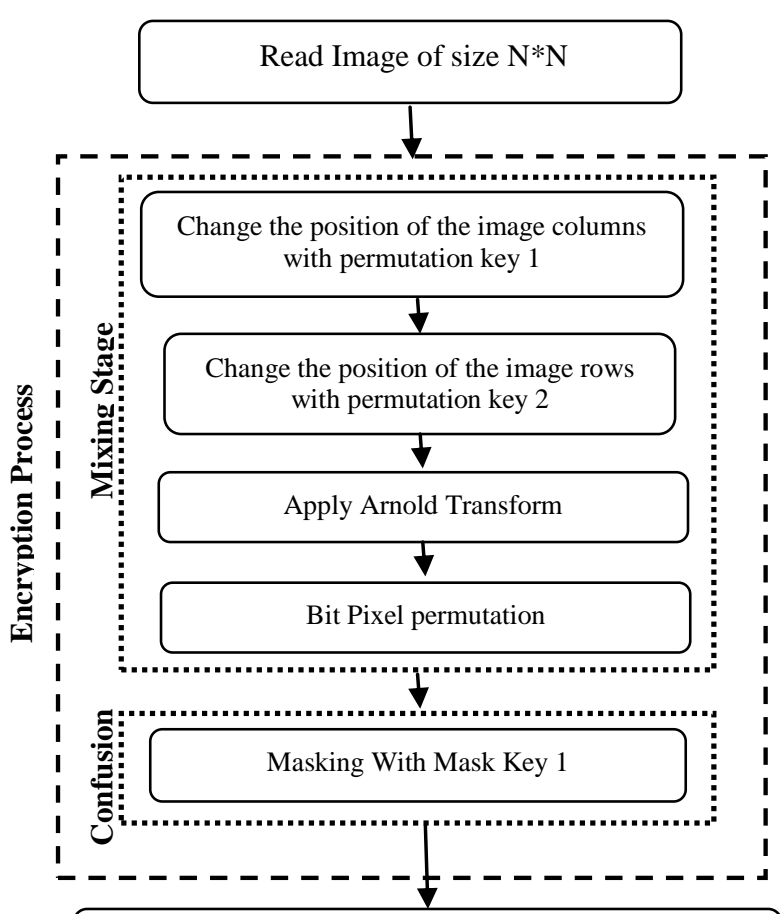

Transmitting the encrypted image in an insecure channel

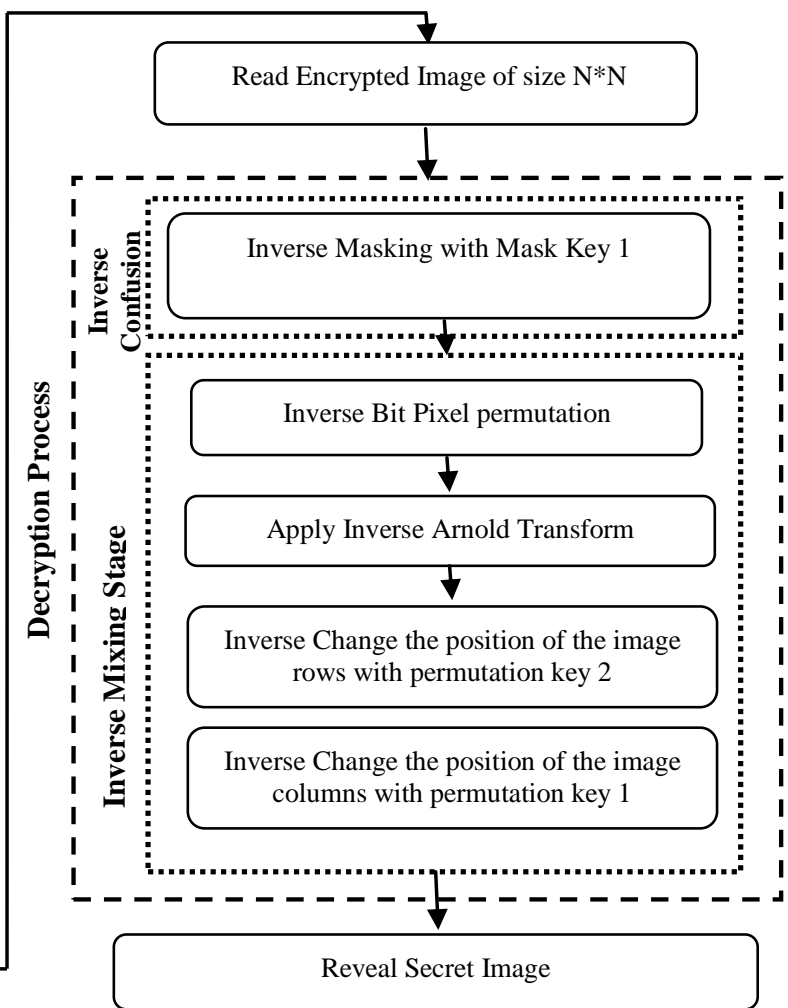

Reveal Secret Image

Fig 1: The General Structure of Encryption and Decryption Processes

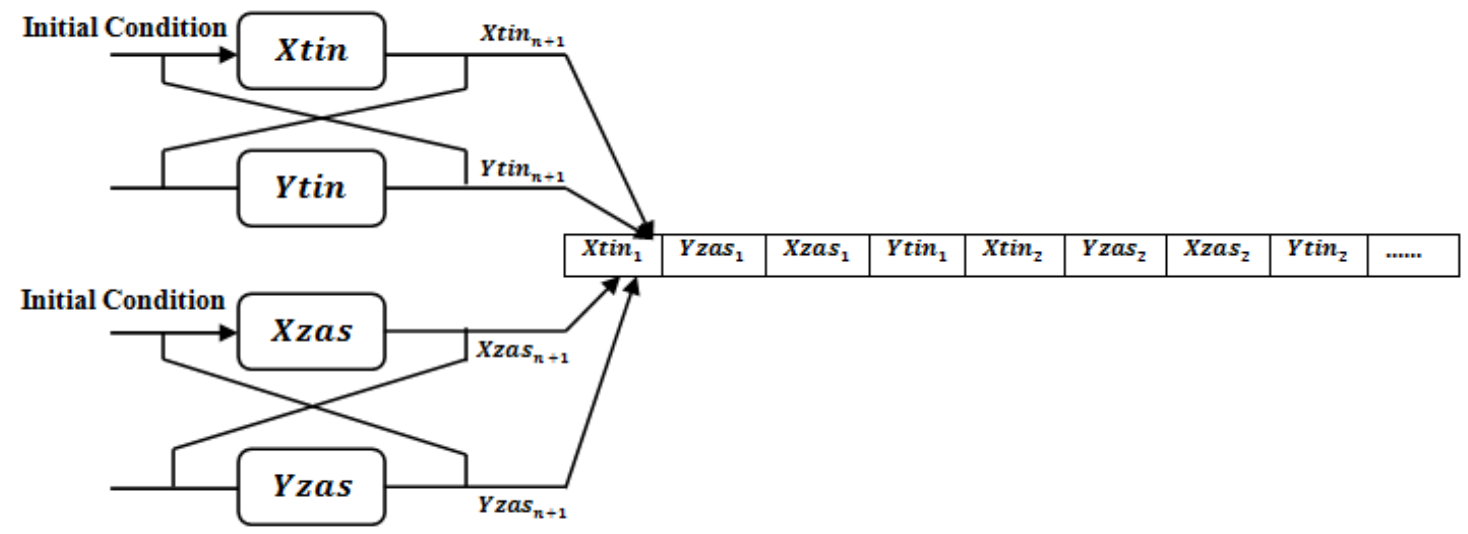

Fig 2: Mixing Keys Generation

\begin{tabular}{|c|c|c|c|c|c|c|c|c|}
\hline \multicolumn{1}{|c|}{ Pixel Value $=65$} \\
\hline Pixel Binary presentation & 0 & 1 & 0 & 0 & 0 & 0 & 0 & 1 \\
\hline Index & 1 & 2 & 3 & 4 & 5 & 6 & 7 & 8 \\
\hline Pixel Binary presentation & 1 & 1 & 0 & 0 & 0 & 0 & 0 & 0 \\
\hline
\end{tabular}

Fig 3: Bit-Pixel Permutation 


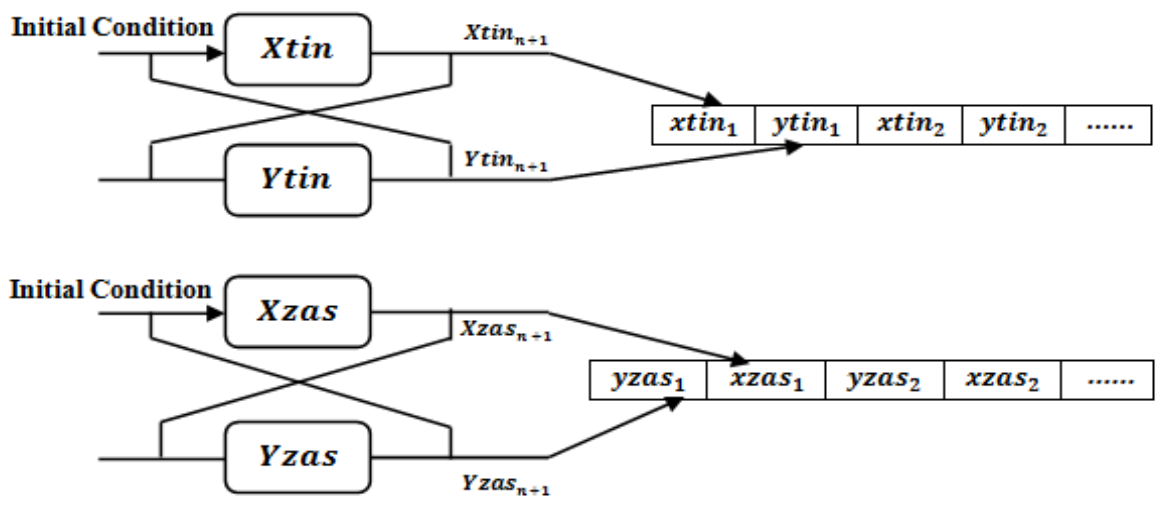

Fig 4: Substitution Keys Generation

\section{SIMULATION RESULT}

The following sub sections are devoted to present and discuss the results of the conducted tests to assess the performance of the proposed systems. Simulation of the proposed image encryption scheme was implemented using Matlab R2013a. six images of size $256 \times 256$ with gray-scale $(0-255)$ were used .The outcomes of the performance of the proposed image encryption are showed as Fig. 5.

\subsection{HISTOGRAM}

The original image and the encrypted image are shown in figure 6 depicting the histograms. The histogram of the encrypted image is nearly uniformly distributed, which can well protect the information of the image to withstand the statistical attack.

\subsection{CORRELATION ANALYSIS}

The reliance of two neighboring variables at a certain bearing is measured by correlation coefficient. The more closely related these two variables are, the closer the correlation coefficient approaches 1 . If they are less closely related, the value of correlation coefficient approaches 0 . The correlation coefficient of adjacent pixels is calculated according to the following equations:

$$
\begin{gathered}
C r=\frac{\operatorname{cov}_{x, y}}{\sqrt{D_{x}} \sqrt{D_{y}}} \ldots .10 \\
\operatorname{cov}_{x, y}=\frac{1}{T} \sum_{i=1}^{T}\left(x_{i}-E(x)\right)\left(y_{i}-E(y)\right) \ldots .11 \\
E(x)=\frac{1}{T} \sum x_{i}, D(x)=\frac{1}{T} \sum\left(x_{i-} E(x)\right)^{2} \ldots .12
\end{gathered}
$$

Where $\mathrm{x}$ and $\mathrm{y}$ are gray values of two adjacent pixels in an encrypted image. 5000 pairs of horizontally were randomly selected, vertically, and diagonally neighboring pixels and calculate the correlation coefficients in three directions separately [7]. The correlation coefficient of the pixel pair is calculated and the result is listed in Table 1 and the distribution is shown in Figure 5.

It is clear that neighboring pixels of the encrypted image have no relationship. Both the correlation coefficients in Table 1 and figure 5 give explanation for the claim that neighboring pixels of the plain image are uncorrelated by the proposed cryptosystem effectively.

\subsection{Entropy}

Average information of the image content is called entropy and should be nearly equals to 8 . The entropy $\mathrm{H}(\mathrm{m})$ of a message source $\mathrm{m}$ can be calculated as:

$$
H(m)=\sum P\left(m_{i}\right) \log _{2} \frac{1}{p\left(m_{i}\right)} \ldots .13
$$

Where $\mathrm{p}(\mathrm{mi})$ represents the probability of symbol $\mathrm{mi}$ and the entropy is expressed in bits. Details of entropy can be found in [8]. The qualities computed in Table 2 are near the perfect value.

\subsection{KEY SPACE ANALYSIS:}

Key space size is the total number of different keys that can be used in the encryption. For a $10^{-10}$ floating point precision, all keys parameters (4 introductory status, 8 control parameters to Transformation keys and 4 introductory status , 8 control parameters to mask key) can take $10^{10}$ possible values. Therefore, the key space comes out as $\left(\left(10^{10}\right)^{24}\right.$, which are big as much as necessary to defend against the brute force-attack.

\subsection{NPCR \& UACI}

The numbers of pixels change rate (NPCR) have also measured to see the influence of changing a single pixel in the original image on the encrypted image by the proposed algorithm. The NPCR measure the percentage of different pixel numbers between the two images. The NPCR is defined by the following equation:

$$
N P C R=\frac{\sum_{i, j} D(i, j)}{M \times N} \times 100 \% \ldots .14
$$

The UACI measures the normalized mean difference rate between the plain image and the encrypted one. UACI is defined by the following formula $[9,10]$ :

$$
\operatorname{UACI}(\mathrm{p}, \mathrm{s})=\left(\frac{1}{n} \sum \frac{\left|p_{i}-S_{i}\right|}{2^{32}-1}\right) \times 100 \% \ldots .15
$$

Table 3, showing The NPCR and UACI values. The obtained NPCR for images encrypted by using the proposed encryption scheme found to be over $99 \%$ showing thereby that the proposed encryption algorithm is very sensitive with respect to small changes in the plaintext. NPCR and UACI prove it is possible to obtain excellent pixel alteration effects. 


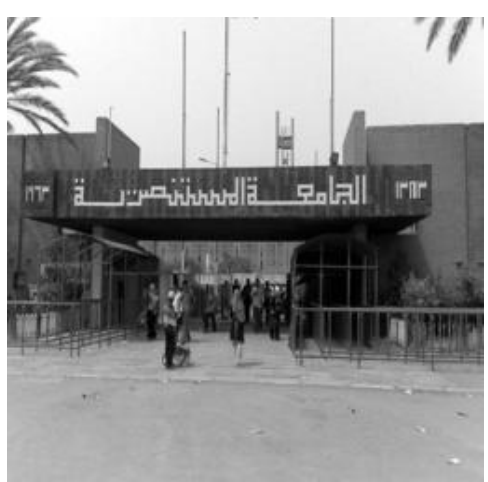

(A) Mustansiria University

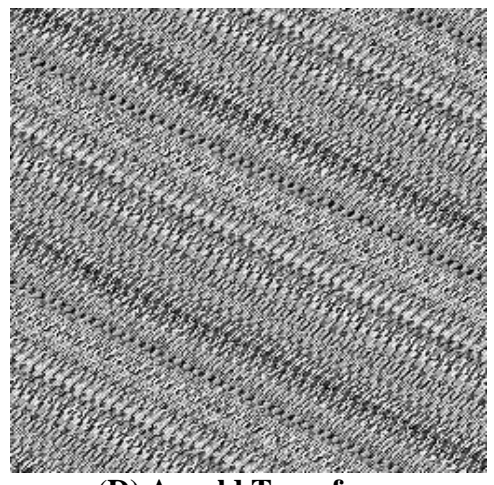

(D) Arnold Transform

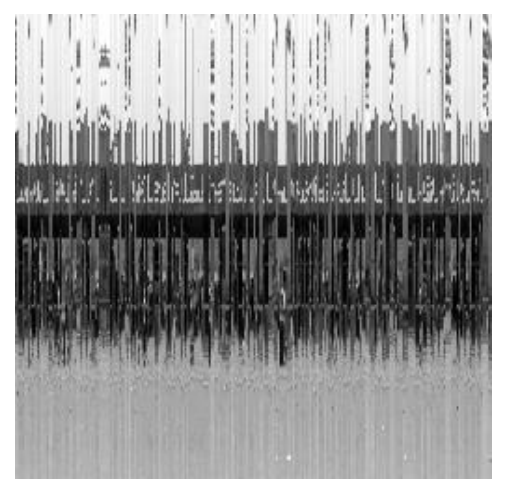

(B) Colum Permutation

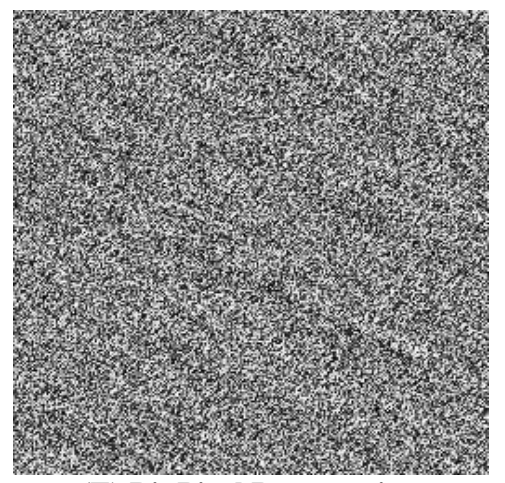

(E) Bit Pixel Permutation

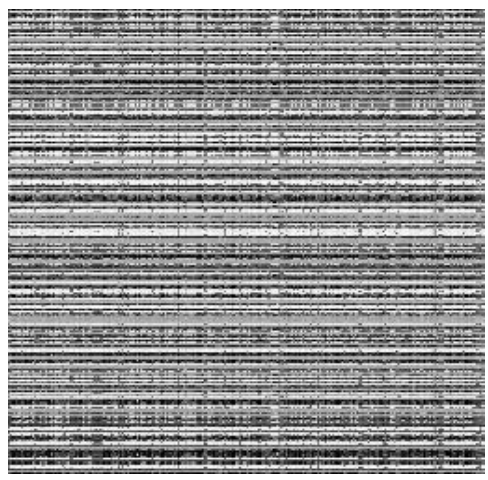

(C) Row Permutation

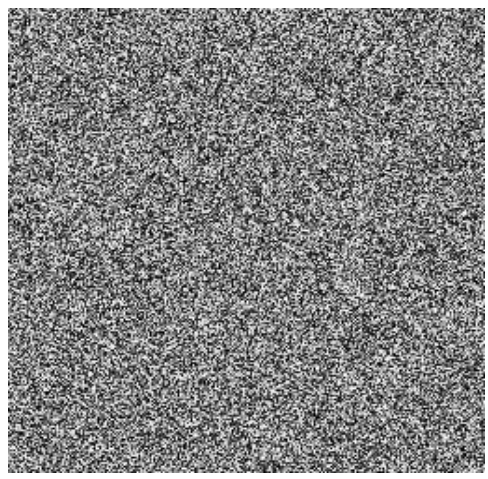

(F) Encrypted Image

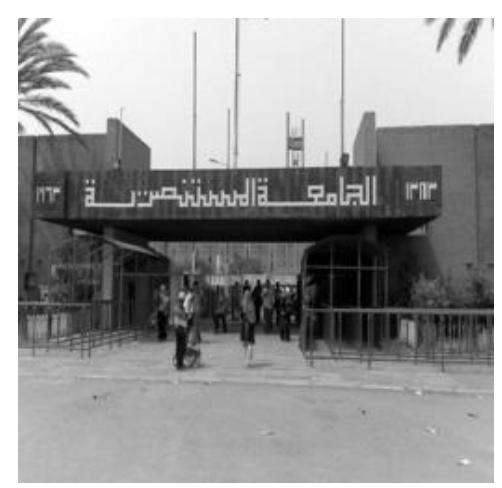

(G) Decrypted Image

Fig 5: Image Encryption Algorithm Result

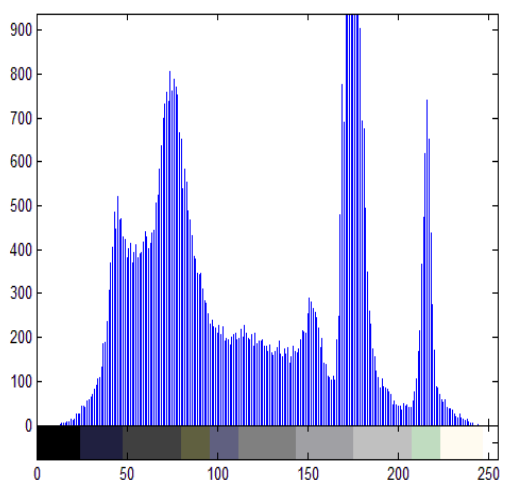

(A) Original

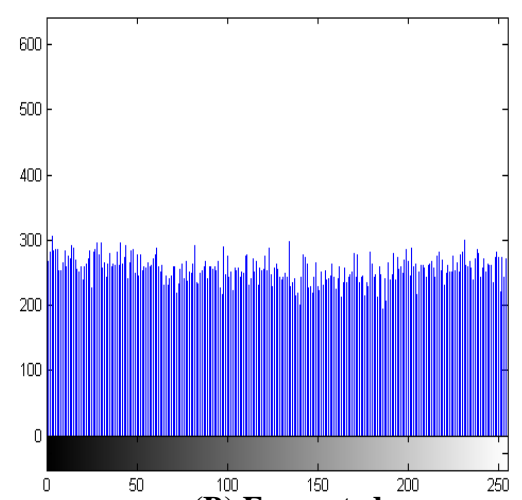

(B) Encrypted

Fig 6: Original Image Histogram and Encrypted 
Table 1: The correlation of adjacent pixels

\begin{tabular}{|c|c|c|c|}
\hline \multirow{2}{*}{ case } & \multicolumn{3}{|c|}{ Original Image } \\
\cline { 2 - 4 } & Horizontal & Vertical & Diagonal \\
\hline 1. & 0.9631 & 0.9759 & 0.9521 \\
\hline 2. & 0.8208 & 0.9144 & 0.8073 \\
\hline 3. & 0.9732 & 0.9588 & 0.9412 \\
\hline 4. & 0.9477 & 0.9296 & 0.8879 \\
\hline 5. & 0.9817 & 0.9904 & 0.9749 \\
\hline 6. & 0.9685 & 0.9680 & 0.9499 \\
\hline \multirow{2}{*}{ Case } & \multicolumn{3}{|c|}{ Encrypted Image } \\
\cline { 2 - 4 } & Horizontal & Vertical & Diagonal \\
\hline 1. & 0.0024 & -0.0017 & 0.0033 \\
\hline 2. & 0.0036 & -0.0006 & -0.0019 \\
\hline 3. & -0.0073 & 0.0116 & -0.0040 \\
\hline 4. & -0.00005 & 0.0001 & -0.0035 \\
\hline 5. & -0.0042 & 0.0192 & -0.0058 \\
\hline 6. & -0.0026 & -0.0038 & 0.0043 \\
\hline
\end{tabular}
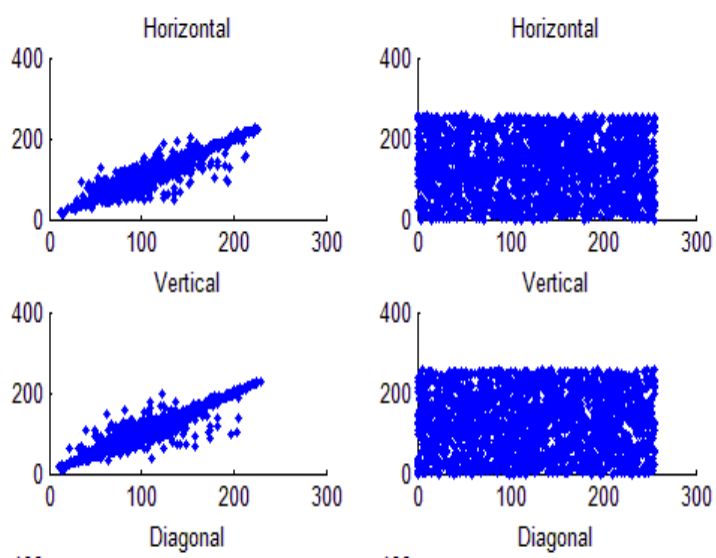

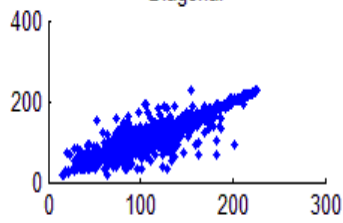

(A

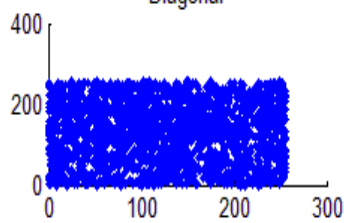

(B)
Fig 7: Correlation of two adjacent pixels: (A) for plain image, and (B) for its encrypted image

\section{CONCLUSIONS}

In this paper, a new algorithm of encryption and decryption of images is proposed. The encryption algorithm use two concepts, i.e., confusion and diffusion which is also called permutation and substitution among the pixels of the gray scale image. To perform the confusion in the plain-image" $s$ pixels, a blend of 2D chaotic map is used which include Tinkerbell map, Zaslavsky map and Arnold Transform. While only Tinkerbell map and Zaslavsky map are used to performing the diffusion.

Experimental tests are carried out with detailed numerical analysis, including key space analysis, statistical attack analysis and differential attack analysis. All the experimental results demonstrate that the proposed encryption algorithm is secure thanks to its bulky key space; it's vastly sensitivity to the encryption keys. For future work 3D chaotic maps can replace the $2 \mathrm{D}$ chaotic maps used in the paper.
Table 2: Entropy value

\begin{tabular}{|c|c|c|}
\hline case & Original Image & Encrypted Image \\
\hline 1. & 7.4197 & 7.9932 \\
\hline 2. & 7.6863 & 7.9930 \\
\hline 3. & 7.5544 & 7.9942 \\
\hline 4. & 7.1333 & 7.9576 \\
\hline 5. & 6.2507 & 7.9108 \\
\hline 6. & 7.6134 & 7.9955 \\
\hline
\end{tabular}

Table 3: NPCR and UACI values

\begin{tabular}{|c|c|c|}
\hline case & NPCR & UACI \\
\hline 1. & 0.99559 & 33.53 \\
\hline 2. & 0.99577 & 33.525 \\
\hline 3. & 0.9958 & 33.48 \\
\hline 4. & 0.9949 & 33.67 \\
\hline 5. & 0.99003 & 33.05 \\
\hline 6. & 0.9962 & 33.48 \\
\hline
\end{tabular}

\section{REFERENCES}

[1] Akhavan A., Samsudin A., Akhshani A., " A novel parallel hash function based on 3D chaoticmap ", EURASIP Journal on Advances in Signal Processing 2013.

[2] Gopalakrishnan T., Ramakrishnan S., Balakumar M., " Image Encryption using Chaos and Parity based Pixel Modification in Permutation", International Journal of Computer Applications® (IJCA), 2014.

[3] Akhshani A. , Behnia S., A. Akhavan, Hassan H. Abu, Hassan Z. , "A novel scheme for image encryption based on 2D piecewise chaotic maps", Optics Communications 283 (2010) 3259-3266.

[4] Goldsztejn A., "Tinkerbell is chaotic", SIAM Journal applied dynamical system, 2011, vol. 10, No. 4, pp. $1480-1501$.

[5] Stoyanov B., Kordov K., "Novel Zaslavsky Map Based Pseudorandom Bit Generation Scheme ", Applied Mathematical Sciences, Vol. 8, 2014, no. 178, 8883 8887.

[6] Tian-gong P., Li Da-yong, " A Novel Image Encryption Using Arnold Cat", International Journal of Security and Its Applications, Vol.7, No.5 (2013), pp.377-386.

[7] Tiegang G., Zengqiang C., "A new image encryption algorithm based on hyper-chaos", Elsevier B.V. All rights reserved 2007 .

[8] Hanchinamani G., Kulakarni L., " A Novel Approach for Image Encryption based on Parametric Mixing Chaotic System ", International Journal of Computer Applications (0975 - 8887) ,Volume 96- No. 11, June 2014

[9] Huang C.K., Liao C.W., Hsu S.L., Jeng ·Y.C., "Implementation of gray image encryption with pixel shuffling and gray-level encryption by single chaotic system ", (C) Springer Science Business Media, LLC 2011.

[10] Chen G., Mao Y., \& Chui, C. K., "A symmetric image encryption scheme based on 3D chaotic cat maps.Chaos", Solitons and Fractals, 21, 749-761, 2004. 